\section{A technique for plastic embedding of mineralised bone}

WHB MA WHINNEY, HA ELLIS From the Department of Pathology, Royal Victoria Infirmary, Newcastle upon Tyne, NE1 4LP

Techniques available for the histological examination of bone vary in their efficiency and reproducibility. For the accurate diagnosis of metabolic bone disease it is necessary to be able to distinguish between mineralised bone and osteoid and this is best accomplished by using sections of undecalcified bone.' If worthwhile quantitative histology is to be undertaken it is desirable that there should be as little shrinkage or other artefact as possible.

For these purposes we initially used Ball's techni$q^{2} e^{2}$ in which undecalcified bone is double embedded in low viscosity nitrocellulose followed by paraffin wax and the integrity of the sections maintained with the aid of Sellotape. However the method has several disadvantages and does not readily permit processing of large specimens such as femoral heads or vertebral bodies.

Present plastic techniques for bone histology commonly use glycol methacrylate or methyl methacrylate as the embedding medium, ${ }^{3-5}$ but both substances have limitations. Although soft tissue components such as osteoid and marrow process well there is commonly cracking and fissuring of the mineralised bone. Furthermore the use of methyl methacrylate involves costly and time consuming preparation of the monomer to remove impurities prior to processing, and partial polymerisation of the monomer. Improvements in the technique were made $^{6}$ with solution of polymethyl methacrylate beads in the monomer in conjunction with the partial polymerisation techniques, resulting in increased consistency.

Glycol methacrylate (JB4, Sorval, HEMA) is extremely useful for smaller bone samples and thin sections. However it gives inconsistent results with blocks much over $10 \mathrm{~mm} \times 10 \mathrm{~mm} \times 3 \mathrm{~mm}$ and mineralised parts of the bone tend to fissure. Glycol methacrylate has also been suspected locally of giving rise to skin sensitivity and to cause dermatitis when used on a regular basis.

During the last 10 years we have overcome many

Accepted for publication 8 June 1983 of these problems by employing the plastic resin Polymaster $1209 \mathrm{AC}^{7}$ to facilitate preparation of our undecalcified bone sections. This particular resin was originally recommended to us by Professor ED Williams. The method we have developed has proved most satisfactory for trans-iliac bone biopsies and large necropsy or surgical specimens of bone. We include a photograph of a mounted section of a complete vertebral body (Fig. 1) and photomicrographs of cortical and cancellous bone from an iliac bone biopsy (Fig. 2a \& b) to illustrate the degree of preservation of tissues which can be expected with this method. Coloured illustrations of the histology of undecalcified bone biopsies processed by this method may be found in a previous publication. ${ }^{8}$ Since we are frequently asked for details of this particular technique we thought it might be helpful to make these more widely available.

Polymaster $1209 \mathrm{AC}$ is a polyester resin incorporating a styrene monomer stabilised by hydroquinone. Addition of an organic peroxide "catalyst" polymerises the resin by addition reactions between unsaturated $\mathrm{C}=\mathrm{C}$ bonds in the polyester polymer, crosslinked with the $\mathrm{C}=\mathrm{C}$ bonds in the styrene. The resulting polymer is extremely inert, forming a hard insoluble plastic that allows most staining reactions commonly used in histology laboratories. In addition it permits the application of more specialised bone staining techniques such as von Kossa, toluidine blue, Masson Goldner, Movat's pentachrome and Aluminon staining for the demonstration of aluminium. It is also suitable for tetracycline labelling studies providing alcohol fixation is used and sections are dehydrated and cleared immediately after cutting. Since the plastic does not itself take up stains it does not intrude in the final preparation and enables sections of suitably sized tissues to be cut down to $2 \mu \mathrm{m}$ in thickness. It is manufactured in large quantities and is of low cost.

\section{Methods}

1 Fix tissues in $10 \%$ buffered formalin or preferably absolute ethanol (Analar) for 2-7 days (ethanol or methanol essential for tetracycline labelling).

2 Dehydrate in cellosolve (alcohol-fixed tissuesfour changes of $3,5,16,24 \mathrm{~h}$; buffered formalinfixed tissues until test shows all buffer salts are removed; see note 2 below).

3 Infiltrate in cellosolve/resin mixtures of increasing resin concentrations of: $75 \% / 25 \%, 50 \% / 50 \%$, $25 \% / 75 \%, 100 \%$, for $24 \mathrm{~h}$ (or longer) in each dilution with agitation. 


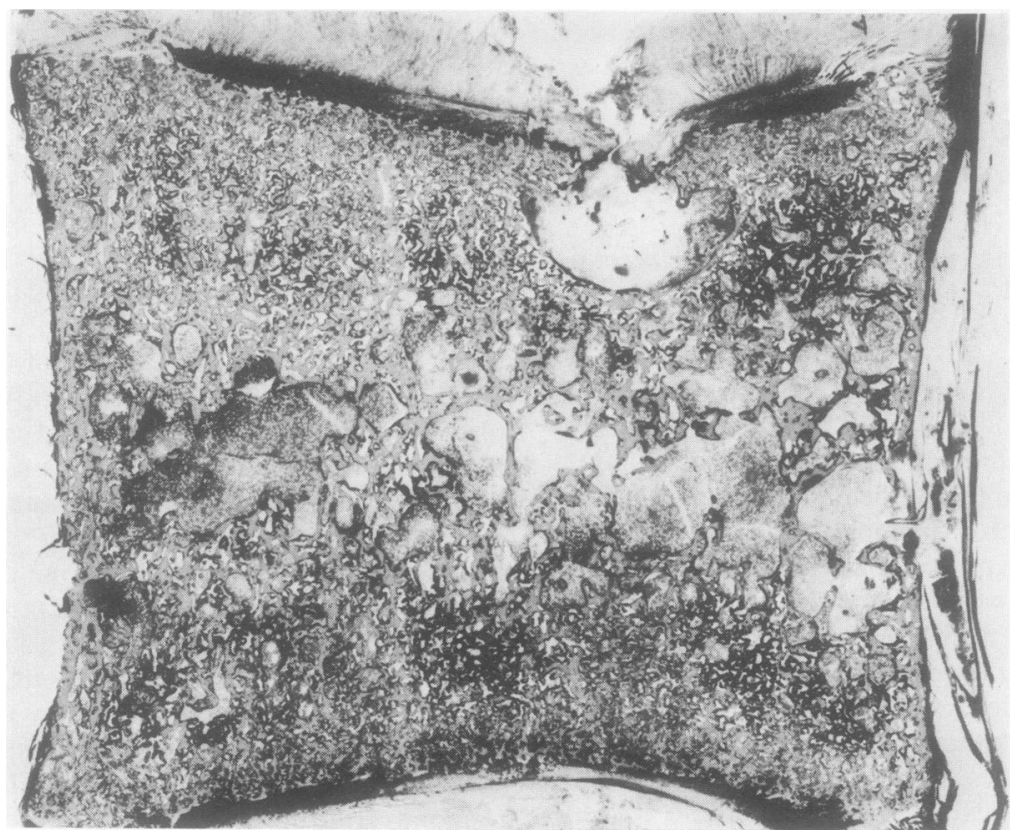

Fig. 1 Undecalcified section of whole vertebral body from a patient with renal bone disease. Note preservation of trabecular architecture in spite of osteosclerosis adjacent to intervertebral discs. There is a coincidental Schmorl's node. Masson Goldner stain (larger patches of osteoid in trabeculae stain red and appear black). Original magnification $\times 4$.

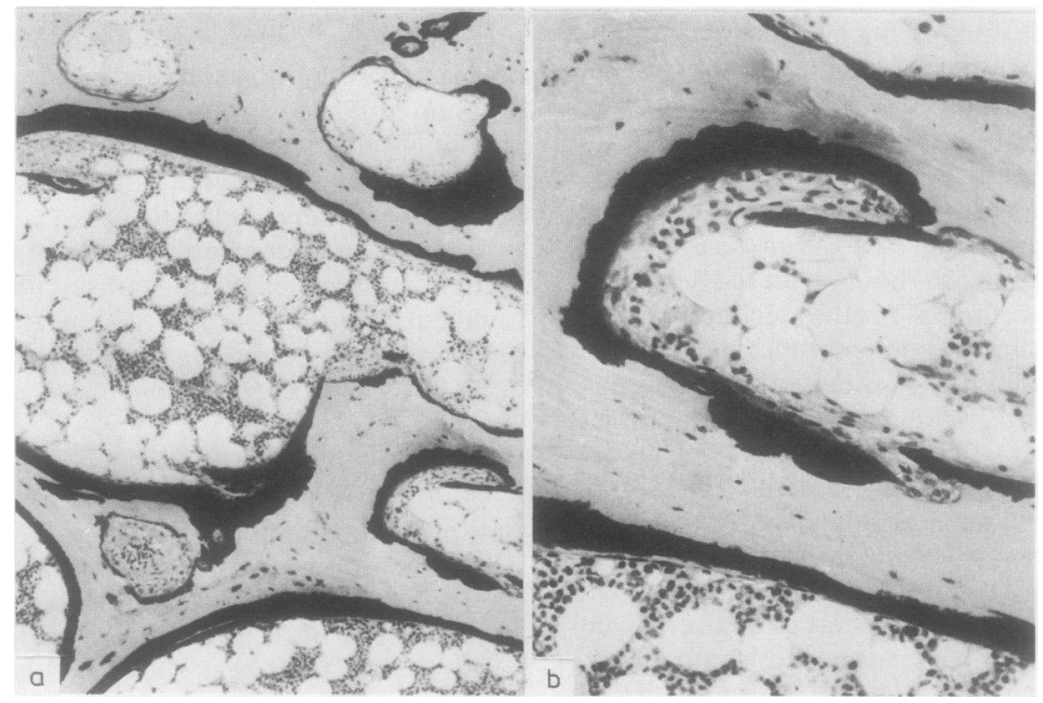

Fig. 2 Photomicrographs of undecalcified section of iliac bone biopsy from a patient with renal bone disease. Note (a) Preservation of architecture of cortical and cancellous bone (b) higher power of part of (a) to show detail of mineralised bone, osteoid and marrow elements. Masson Goldner stain (mineralised bone stains green, appears pale; osteoid stains red, appears black). Original magnifications (a) $\times 70$; (b) $\times 180$. 
4 Infiltrate in three changes of $24 \mathrm{~h}$ each at $37^{\circ} \mathrm{C}$ and $700 \mathrm{~mm}$ vacuum in:

Resin

Dibutyl phthalate

$95 \%$

$5 \%$

5 Infiltrate for $8 \mathrm{~h}$ in agitator in:

Resin

Dibutyl phthalate

$95 \%$

$5 \%$

Butanox 50 "catalyst"

$1 \%$ hydroquinone in ethanol

$1 \%$

$1 \%$

6 Embed in siliconised glass mould and leave in waterbath heatsink at room temperature overnight (or until polymerised) (see note 3 below).

7 Harden in $56^{\circ} \mathrm{C}$ oven for $48 \mathrm{~h}$.

8 Remove block from mould and trim off excess resin with a bandsaw and sander.

9 Rough trim blocks on microtome and cut sections (routinely $6 \mu \mathrm{m}$ ) using tungsten carbide knife (Jung K, HK2 knife, or Jung Polycut) and 70\% ethanol as a lubricant.

10 Sections are stained free floating through staining solutions, dehydrated, cleared and mounted in DPX.

\section{Notes}

1 The time taken for processing varies depending on the size of the tissue. Times as given above are suitable for blocks measuring from $6 \mathrm{~mm} \times 12 \mathrm{~mm}$ $\times 3 \mathrm{~mm}$ up to $20 \mathrm{~mm} \times 30 \mathrm{~mm} \times 3 \mathrm{~mm}$ and collectively require three weeks to complete. For larger blocks up to and including $5 \mathrm{~mm}$ thick slices of femoral head or vertebral body it is found that doubling the recommended times is suitable.

The process can be safely shortened for small (2 mm smallest dimension $\times 5 \mathrm{~mm}$ largest dimension) alcohol fixed blocks by dehydrating in cellosolve overnight, reducing cellosolve/resin infiltration times to 2 hours in each dilution, and 16 hours (overnight) in resin/dibutyl phthalate under vacuum. Following the recommended times for infiltration in "catalyst" and polymerisation the block can be ready for cutting after 5 days.

2 Polyester resin processing requires that there be no water left in the tissue prior to infiltration with resin. Alcohol fixation is useful in meeting this requirement particularly with biopsy trephines, however some tissue blocks will still be received in formalin fixatives in most laboratories. Tests have shown that buffer salts in formalin fixatives continue to leave the specimen as it is dehydrated in cellosolve. The buffer salts can easily be detected by adding $1 \mathrm{ml}$ of $2 \%$ aqueous silver nitrate to $2 \mathrm{ml}$ of the used dehydrating cellosolve. A faint white cloudiness in the fluid can be seen with concentrations of buffer salts down to that associated with $0.2 \%$ water. Use of this test with buffered formalin fixed tissues of all sizes including femoral heads and longitudinal sections of vertebrae has ensured consistent resin processing.

3 Use of a heatsink at the early polymerisation stage prevents bubble formation due to vaporisation of cellosolve by the exothermic polymerisation reaction.

\section{References}

' Ellis HA. Metabolic bone disease. In: Anthony PP, MacSween RNM, eds. Recent advances in histopathology. Edinburgh: Churchill-Livingstone, 1981:185-202.

${ }^{2}$ Ball J. A simple method of defining osteoid in undecalcified sections. J Clin Pathol 1957;10:281-2.

${ }^{3}$ Yaeger JA. Methacrylate embedding and sectioning of calcified bone. Stain Technol 1958;33:229-39.

4 Bennett HS, Wyrick AD, Lee SW, McNeill JH. Science and art in preparing tissues embedded in plastic for light microscopy, with special reference to glycol methacrylate, glass knives and simple stains. Stain Technol 1976;51:71-97.

s te Velde J, Burkhardt R, Kleiverda K, Leenheers-Binnendijk L, Sommerfeld W. Methyl-methacrylate as an embedding medium in histopathology. Histopathology 1977;1:319-30.

${ }^{6}$ Difford J. A simplified method for the preparation of methyl methacrylate embedding medium for undecalcified bone. $J$ Med Lab Technol 1974;31:79-81.

7 Polymaster 1209 AC is supplied by Bondaglass-Voss Ltd, 158164 Ravenscroft Road, Beckenham, Kent.

' Pierides AM, Edwards WG, Cullum UX, McCall JT, Ellis HA. Hemodialysis encephalopathy with osteomalacic fractures and muscle weakness. Kidney Int 1980;18:115-24.

Requests for reprints to: Dr HA Ellis, Department of Pathology, Royal Victoria Infirmary, Newcastle upon Tyne NE1 4LP, England. 